\title{
Upstream of EMS Overutilization: The Tufts First Aid Kit Project
}

\author{
Justin Zaslavsky, EMT; Carly Eiduson, EMT; Isaiah Smolar, AEMT; \\ Andrew Pettit, EMT
}

\section{POSTER PRESENTATION ABSTRACT}

Introduction: In 2016, EMTs from Tufts Emergency Medical Services (TEMS) noticed an under-preparedness amongst first-year students to handle minor illness and injury. The EMTs observed that first-year students often sought expensive and resource-intensive EMS and ED care for minor conditions that could be managed by either a general practitioner or themselves, demonstrating a limited understanding of the differences between emergency and non-emergency resources available to the Tufts community while putting an unnecessary - and potentially dangerous - strain on both university and municipal services. Multiple instances were also seen of first-year students' requesting an ambulance for medical supplies (eg, thermometers or ice packs) with no intention of seeking definitive care, demonstrating students' not having sufficient equipment for self-treatment. Program Development \& Implementation: TEMS — alongside Tufts Health Promotion and Prevention—funded, created, and distributed first aid kits to all first-year students, which included personalized informational packets to address these two problematic observations. The project has since expanded to involve more of the first-year experience, including the implementation of an informational video that was developed and shown in Fall 2017 during first-year orientation. Program Evaluation: A survey was distributed to students of all class years, which demonstrated differences between students who did and did not receive a first aid kit surrounding attitudes toward the various medical services offered at Tufts. The survey also indicated differences in student's reported help-seeking behavior. Additionally, a chart review was completed, demonstrating that a smaller portion of first-year students refused transport after evaluation compared to second-year students $(13.7 \%$ vs. $25 \%$, p $<.05)$, possibly indicating a higher threshold for requesting EMS. Discussion/Conclusion: By providing first aid kits and information, collegiate EMS agencies are able to take a public health role within the communities they serve. TEMS hopes to continue this project by obtaining secure funding and constantly evaluating to ensure that students receive necessary supplies in the most cost and energy efficient way.

Author Affiliations: Emergency Medical Services, Tufts University, Medford, MA, USA

Address for Correspondence: Justin Zaslavsky, EMT. E-mail: Justin. Zaslavsky@tufts.edu

Conflicts of Interest/Funding Sources: By the JCEMS Submission Declaration Form, all authors are required to disclose all potential conflicts of interest and funding sources. The authors declared that they have no conflicts of interest. The authors declared that they received funding from Tufts University Emergency Medical Services, Tufts University Health Promotion and Prevention, and Tufts University Health Services for the program and/ or research described in this abstract.

Ethical Compliance: The authors attest that the research associated with this abstract was conducted in accordance with the JCEMS Ethics Guidelines.

Submission History: Received January 15, 2018; accepted for presentation and publication February 22, 2018.

Poster Presentation: This abstract was presented as a poster at the Academic Poster Session of the $25^{\text {th }}$ Annual Conference of the National Collegiate
Emergency Medical Services Foundation; February 24, 2018; Philadelphia, PA, USA. The authors received the First Place Award for the Best Poster Presentation Competition. The poster is available for download on the JCEMS website.

Published Online: April 24, 2018

Published in Print: August 13, 2018 (Volume 1: Supplemental 1)

Reviewer Information: In accordance with JCEMS editorial policy, poster presentation abstracts undergo double-blind peer-review by at least two reviewers (JCEMS Editorial Board members and/or independent reviewers) prior to acceptance for presentation and publication. JCEMS thanks the anonymous reviewers who contributed to the review of this work.

Copyright: (C) 2018 Zaslavsky, Eiduson, Smolar, \& Pettit. This is an open access abstract distributed under the terms of the Creative Commons Attribution 4.0 International (CC BY 4.0) License, which permits unrestricted use, distribution, and reproduction in any medium, provided the original author and source are credited. The full license is available at: https://creativecommons.org/licenses/by/4.0/

Electronic Link: https://doi.org/10.30542/JCEMS.2018.01.S1.01 